\title{
Perception of static respiratory forces in young and old subjects
}

\author{
ANDREW HARVER and HARRY KOTSES \\ Ohio University, Athens, Ohio
}

\begin{abstract}
Respiratory sensations were measured by magnitude production in 15 young (23-33 years) and 15 old (61-85 years) healthy males to compare exponents for static respiratory muscle forces in the two age groups. Perceptions of static inspiratory and expiratory forces were scaled both at the end of normal expiration and at lung volume extremes. Exponents for young and old groups did not differ, and were independent of lung volume. The average exponent for perceived respiratory force was 1.7 .
\end{abstract}

Sensations surrounding respiratory efforts against closed airways, breathing against mechanical loads, and inspiring different tidal volumes have been examined during the past decade with psychophysical magnitude scaling procedures (see Zechman \& Wiley's, 1986, review). Perceived magnitude in the first two situations is related directly to tension generated by actively contracting respiratory muscles, and in the third to thoracic displacement (e.g., Altose, Dimarco, Gottfried, \& Strohl, 1982; Killian, Bucens, \& Campbell, 1982; Tack, Altose, \& Cherniack, 1983; Zechman \& Wiley, 1986). Despite the definition of such relationships, the sensory mechanisms mediating these experiences remain uncertain.

Comparisons between young ( $<30$ years) and old $(>60$ years) subjects show that chronological age affects breathing sensations and that age-group differences in power function exponents depend on the breathing sensation scaled: Although exponents for inspiratory efforts against closed airways are comparable in young and old subjects, exponents for breathing against added mechanical loads are less, and those for production of graded inspiratory volumes are greater, in older than in younger subjects (A1tose, Leitner, \& Cherniack, 1985; Tack, Altose, \& Cherniack, 1981, 1982, 1983). Similarities in the processing of signals related to tension or muscle force in young and old subjects have been hypothesized to account for the lack of difference in exponents for static efforts; agerelated differences in relative integration by higher brain centers of signals related to both tension or muscle force and displacement have been hypothesized to account for the difference in exponents for added loads and for inspired volumes (Altose et al., 1985; Tack et al., 1983).

The research described in this report was supported, in part, by Grant 27402 from the Division of Lung Diseases, National Heart, Lung, and Blood Institute. It was submitted by A.H. to the Department of Psychology, Ohio University, in partial fulfillment of the requirements for the PhD degree. The authors thank John C. Baird, Donald A. Mahler, and Martha Teghtsoonian for many helpful comments. Requests for reprints should be sent to Andrew Harver, Department of Physiology, Dartmouth Medical School, Hanover, NH 03756.
Possibly, the pattern of exponents exhibited by young and old groups is influenced also by flow-related characteristics of breathing sensations: Perception of inspiratory efforts against closed airways is examined under static (i.e., no-flow) conditions, whereas perception of added loads and inspired volumes is examined under dynamic (i.e., flow-dependent) conditions.

Despite these hypotheses, relatively little evidence has accumulated to support the contention that perception of static forces is not affected by age. Such evidence is limited to examination of inspiratory forces produced against a closed airway following normal expiration (Tack et al., 1983). Neither the effects of airflow direction nor the effects of lung volume have been considered in this context. The lung volume at which static forces are scaled may influence exponents in young and old subjects differentially because age-related changes in respiratory physiology include hyperinflation, increased chest wall stiffness, and decreased lung elasticity (Cherniack \& Brown, 1965; Mahler, 1983; Mittman, Edelman, Norris, \& Shock, 1965).

We examined effects of chronological age on the perception of static respiratory forces by comparing exponents between young and old subjects for static inspiratory and expiratory forces produced both at the end of a normal expiration (i.e., at functional residual capacity) and at the extremes of lung volume (i.e., at residual volume and at total lung capacity). All subjects participated also in magnitude scaling tasks of visual line length to provide control information about the perceptual ability of subjects on scaling tasks unrelated to respiration.

\section{METHOD}

\section{Subjects}

Fifteen male graduate students between the ages of 23 and 33 years $($ mean $=26.07$, range $=23-33$ ) and 15 male retired faculty members between the ages of 61 and 85 years (mean $=69.8$, range $=61-85$ ) participated. All participants were nonsmokers with no known history of either pulmonary or cardiovascular disease; all were unfamiliar with the purpose of the investigation. 


\section{Apparatus}

Lung volumes prior to the magnitude production of static respirations and forced expirations were recorded with a Godart watersealed spirometer (Model 16000).

Pressure was sampled near the mouth from a two-way valve by a differential pressure transducer (Validyne, MP45-32-871, range $\pm 100 \mathrm{cmH}_{2} \mathrm{O}$ ). The pressure signal was conditioned by a carrier demodulator (Validyne, CD15), recorded with a Beckman Type 9806A coupler, and displayed on one channel of a Beckman Type RM Dynograph recorder.

\section{Procedure}

Each subject participated in two experimental sessions completed, on average, within 8 days. At each session, pulmonary evaluations were conducted by measuring lung function, maximal inspiratory and expiratory pressures, and relaxation pressures. Evaluation of lung function involved analysis of the volume and timing components of a rapid and forceful expiration. Maximal inspiratory and expiratory pressures were obtained by encouraging subjects to exert maximal inspiratory and expiratory efforts, respectively, against a closed airway at functional residual capacity (i.e., the end of a normal expiration). Relaxation pressures at residual volume (i.e., the end of a maximal expiration) and at total lung capacity (i.e., the end of a maximal inspiration) were obtained by instructing subjects to relax momentarily with the glottis open against a closed valve.

Following these initial assessments, the subject participated in either a magnitude estimation (ME) or magnitude production (MP) task. In both cases, he engaged in the direct scaling of line length. For the ME task, the subject provided numerical estimates of visual length for 10 lines, varying in length between 5 and $250 \mathrm{~mm}$, displayed separately on nonruled $81 / 2 \times 11$ in. sheets of paper. The set of lines was presented twice to each subject in random order with the restriction that the line of median length was presented at the start of each sequence. The subjects were instructed to "call the first stimulus, which will be of median length, any number that seems appropriate," and to assign successive numbers in such a way that "they reflect your subjective impression."

For the MP task, the subject performed the reverse task; he drew lines of varying length for each of 10 values ranging between 5 and 250 . Each response was made on a separate sheet of $81 / 2 \times 11$ in. paper. The set of values was presented to each subject twice in random order with the restriction that the median value was presented at the start of each sequence. The order in which the two tasks were administered to all subjects was counterbalanced between the two sessions.

After the line length task, the subject engaged in a second MP task. In this case, he produced either inspiratory or expiratory respiratory forces against a closed airway to match particular numbers. The subject was given the following instructions (adapted from Stevens, 1975, p. 30): "You will be presented with a series of numbers in irregular order. Your task is to tell how different they seem by generating static forces with your muscles of respiration. The first number will be about the middle value; make the first force of any magnitude that seems appropriate to you. Then produce successive forces in such a way that they reflect your subjective impression. Try to generate the forces only with the muscles of respiration. Try to make each force match the magnitude of the number as you perceive it."

Following these instructions, static forces were produced for each of nine values ranging between 20 and 200 . The set of values was presented to each subject twice in random order at each of two lung volumes with the following restrictions: The median value was presented at the start of each sequence, and both sequences were completed at one lung volume before the other was presented. Perceived muscular force of static inspirations was scaled at both functional residual capacity (FRC) and residual volume (RV); perceived muscular force of static expirations was scaled at both FRC and total lung capacity (TLC). Prior to each effort, the subject took two or three normal breaths on the spirometer and then inhaled or exhaled to the target lung volume. At this point, the experimenter occluded the breathing circuit near the mouth by turning a stopcock, which remained closed for the duration of a single static effort. The subjects performed all maneuvers while seated, with a nose clamp in place.

The subjects produced only static inspirations or static expirations at each experimental session; the order in which the respiratory scaling tasks were completed was counterbalanced between the two sessions.

\section{RESULTS}

\section{Data Scoring and Analyses}

Magnitude scaling of visual length. Individual exponents for perceived line length were obtained by computing geometric means of estimates for each line length value and solving Stevens's (1975) power function equation using the method of least squares. Exponents were compared between groups with unpaired $t$ tests.

Magnitude production of static respirations. Pressure produced at FRC reflects only tension due to actively contracting respiratory muscles, whereas tension produced at lung volumes both above and below FRC reflects not only (active) tension resulting from contracting respiratory muscles, but also (passive) tension resulting from net chest wall and lung recoil forces (Altose et al., 1982; Cherniack, 1983). Peak pressures recorded at TLC and at RV were adjusted, therefore, to reflect only active tension for each subject by deriving the difference between peak pressures and recoil pressures recorded at maximal lung volumes. The estimate of recoil pressure used in this context was relaxation pressure. Individual exponents between perceived magnitude and muscle force were obtained for each task by solving Stevens's power function using the method of least squares and were compared between groups of subjects. Differences between the exponents computed for static inspirations scaled at FRC and those scaled at RV, and differences between exponents for static expirations scaled at FRC and TLC, were also tested separately for younger and older subjects with paired $t$ tests. Geometric means of the responses for all

Table 1

Mean Pulmonary Function in Young and Old Subjects

\begin{tabular}{|c|c|c|c|c|c|c|}
\hline \multirow[b]{2}{*}{ Subjects } & \multicolumn{2}{|c|}{ FVC } & \multicolumn{2}{|c|}{ FEV, } & \multicolumn{2}{|c|}{$\mathrm{FEF}_{23-75}$} \\
\hline & Mean & $S D$ & Mean & $S D$ & Mean & $S D$ \\
\hline \multicolumn{7}{|c|}{ Session 1} \\
\hline Young & 101.23 & 7.99 & 99.15 & 12.61 & 99.73 & 26.71 \\
\hline Old & 102.63 & 14.36 & 109.37 & 11.46 & 135.17 & 34.10 \\
\hline \multicolumn{7}{|c|}{ Session 2} \\
\hline Young & 97.17 & 7.87 & 97.29 & 12.51 & 102.32 & 32.94 \\
\hline Old & 99.35 & 13.57 & 109.14 & 11.48 & 134.27 & 48.72 \\
\hline
\end{tabular}

Note-FVC $=$ forced vital capacity; FEV $_{1}=$ forced expiratory volume in $1 \mathrm{sec} ; \mathrm{FEF}_{25-75}=$ maximal midexpiratory flow rate. Values are percent predicted (from Kory, Callahan, Boren, \& Syner, 1961). 
Table 2

Mean Maximal Pressures Generated at Functional Residual Capacity by Young and Old Subjects

\begin{tabular}{|c|c|c|c|c|}
\hline \multirow[b]{2}{*}{ Subjects } & \multicolumn{2}{|c|}{ Inspiration } & \multicolumn{2}{|c|}{ Expiration } \\
\hline & Mean & $S D$ & Mean & $S D$ \\
\hline \multicolumn{5}{|c|}{ Session 1} \\
\hline Young & -74.92 & 23.82 & 82.40 & 25.65 \\
\hline Old & -73.42 & 27.40 & 92.18 & 25.23 \\
\hline \multicolumn{5}{|c|}{ Session 2} \\
\hline Young & -82.12 & 23.54 & 83.75 & 28.29 \\
\hline Old & -76.41 & 26.63 & 87.05 & 26.24 \\
\hline
\end{tabular}

Note-Values are given in $\mathrm{cmH}_{2} \mathrm{O}$.

Table 3

Mean Relaxation Pressures Exhibited by Young and Old Subjects

\begin{tabular}{lccccc}
\hline & \multicolumn{2}{c}{ Residual Volume } & & \multicolumn{2}{c}{ Total Lung Capacity } \\
\cline { 2 - 3 } Subjects & Mean & $S D$ & & Mean & $S D$ \\
\hline Young & -5.73 & 4.60 & 6.32 & 5.76 \\
Old & -1.75 & 1.25 & 2.80 & 2.60 \\
\hline
\end{tabular}

Note-Values are given in $\mathrm{cmH}_{2} \mathrm{O}$.

subjects were displayed by plotting logarithmic psychological magnitude as a function of logarithmic pressure. Absolute levels of pressure generated by subjects for each task were examined separately with analyses of variance.

Lung volume changes. Changes in lung volume from FRC achieved by younger subjects prior to the production of static maneuvers at RV and at TLC were compared with those exhibited by older subjects. To accomplish this, expiratory reserve volume (the volume of air expelled from FRC to RV) and inspiratory capacity (the volume of air inspired from FRC to TLC) were measured and compared separately between groups of subjects with unpaired $t$ tests.

\section{Pulmonary Evaluations}

Spirometric measures (determined according to the recommendations of Kory, Callahan, Boren, \& Syner, 1961) of all subjects, summarized in Table 1 in terms of percent predicted, were within normal limits. Mean maximal pressures generated at FRC by both young and old subjects are presented in Table 2 . There were no reliable differences in these levels as a function of age. Relaxation pressures are summarized in Table 3 . There were reliable differences as a function of age for pressures recorded at $\mathrm{RV}[t(28)=3.24, p<.05]$ and at TLC $[t(28)=2.16, p<.05]$.

The results of the spirometric evaluations indicate that lung function was not impaired in either group. Maximal respiratory pressures were comparable between groups of subjects. On the other hand, older subjects exhibited diminished recoil forces at both RV and TLC.

\section{Magnitude Scaling of Visual Length}

Young and old subjects made similar judgments about line length; mean exponents are given in Table 4. The mean exponent for the magnitude estimation task was 0.84 for the young subjects and 0.90 for the old $[t(28)=-1.5$, $p>.05]$. The mean exponent for the magnitude production task was 1.12 for the young subjects and 1.17 for the old $[t(28)=-0.72, p>.05]$. These results are consistent with previous findings that young and old individuals do not differ in terms of exponents for line length (e.g., Tack et al., 1981).

\section{Magnitude Production of Static Inspirations}

Age. The mean exponent for muscular force of static inspiration produced at FRC did not differ between young and old subjects [1.95 and 1.51 , respectively; $t(28)=$ $1.84, p>.05]$. Geometric mean values of pressure produced at FRC as a function of number assigned are shown in Figure 1.

The mean exponent for muscular force of static inspiration produced at RV also did not differ between young and old subjects $[2.06$ and 1.52 , respectively; $t(28)=$ $1.45, p>.05]$. Muscular force in this condition was defined as the difference between peak and relaxation pressures at RV, a measure that specifies pressure due to actively contracting respiratory muscles. Geometric mean values of pressure produced at RV as a function of number assigned are shown in Figure 2.

Lung volume. For both young and old groups, exponents for static inspirations scaled at FRC were not different from exponents for static inspirations scaled at RV.

Table 4

Exponents Obtained for Young and Old Subjects

\begin{tabular}{|c|c|c|c|c|c|}
\hline \multirow[b]{3}{*}{ Psychophysical Scaling Task } & \multicolumn{4}{|c|}{ Measured Exponent } & \multirow[b]{3}{*}{$t$} \\
\hline & \multicolumn{2}{|c|}{ Young } & \multicolumn{2}{|c|}{ Old } & \\
\hline & Mean & $S D$ & Mean & $S D$ & \\
\hline Magnitude estimation of line length & 0.84 & 0.12 & 0.90 & 0.12 & -1.50 \\
\hline Magnitude production of line length & 1.13 & 0.13 & 1.17 & 0.23 & -0.72 \\
\hline Magnitude production of static inspirations at FRC & 1.95 & 0.62 & 1.51 & 0.59 & 1.84 \\
\hline $\begin{array}{l}\text { Magnitude production of static inspirations at RV } \\
\text { adjusted for measured relaxation pressure }\end{array}$ & 2.06 & 0.92 & 1.52 & 1.12 & 1.45 \\
\hline Magnitude production of static expirations at FRC & 1.84 & 0.90 & 1.23 & 0.87 & 1.32 \\
\hline $\begin{array}{l}\text { Magnitude production of static expirations at TLC } \\
\text { adjusted for measured relaxation pressure }\end{array}$ & 1.61 & 0.69 & 1.72 & 0.89 & -0.36 \\
\hline
\end{tabular}

Note-FRC = functional residual capacity; RV = residual volume; $T L C=$ total lung capacity.

All $t$ values not significant $(p>.05)$. 


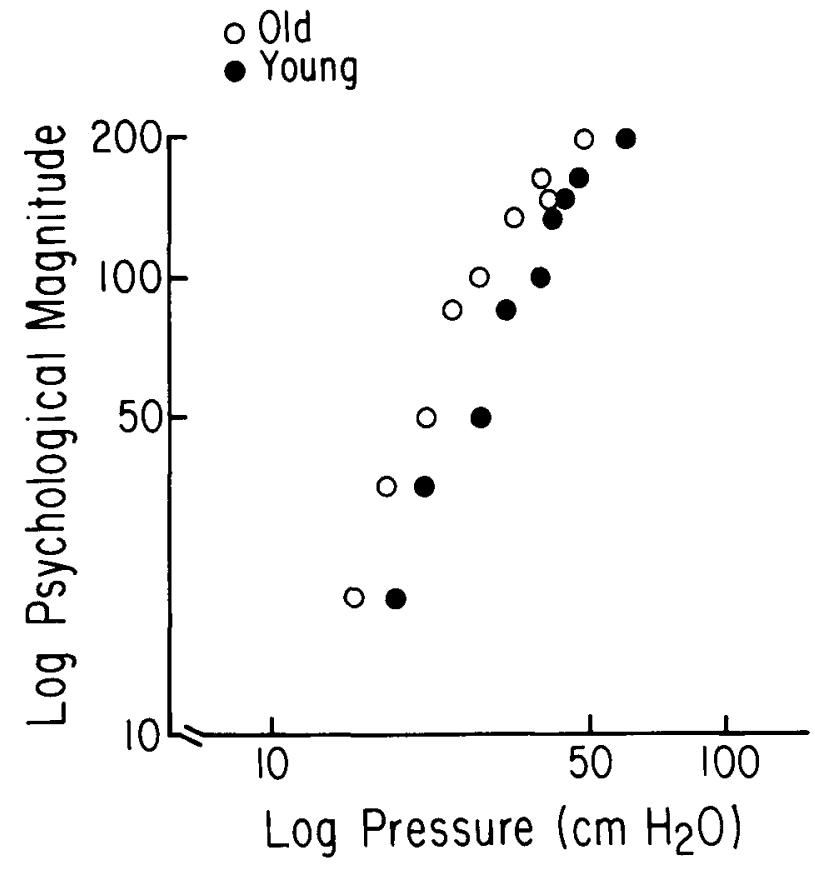

Figure 1. Relationship between psychological magnitude assigned by the experimenter and static inspirations at FRC produced by young and old subjects. Coordinates are logarithmic.

Paired $t$ tests conducted within groups resulted in nonsignificant terms.

Inspiratory pressure. The range of pressures produced during magnitude scaling was comparable between young and old subjects. Although Figures 1 and 2 show an apparent difference between groups in absolute levels of pressure produced during magnitude scaling, the difference is not reliable. However, absolute levels increased as a function of psychological magnitude. This observation was supported by a reliable effect for psychological magnitude $[F(8,244)=71.36]$ revealed by an analysis of variance testing effects due to age and psychological magnitude, with repeated measures on the second factor (pressures generated at FRC), and by a reliable effect for psychological magnitude $[F(8,244)=74.73]$ revealed by an analogous analysis of variance for pressures generated at RV. Neither analysis showed a reliable effect for age.

\section{Magnitude Production of Static Expirations}

Age. The exponents for muscular force exerted in static expirations produced at FRC did not differ between young and old subjects. The mean exponent was 1.84 in the young subjects and 1.23 in the old subjects $[t(28)=1.32$, $p>$.05]. Functions relating the geometric means of static forces produced to match assigned magnitudes are shown in Figure 3.

Young and old subjects also did not differ for muscular force exerted in static expirations at TLC. The mean exponent for the young group was 1.61; for the old group, it was 1.72 . The difference is not reliable $[t(28)=-0.36$, $p>.05]$. The relationship between the geometric means of static forces produced to match assigned magnitudes is shown in Figure 4.

Lung volume. Exponents for static expirations were not influenced differentially by lung volume in either the young or the old group.

Expiratory pressure. Despite the appearance of Figure 3, no differences between groups in absolute levels of pressure produced during magnitude scaling were observed. However, these levels increased as a function of psychological magnitude in both tasks. These observations were supported by reliable effects for assigned numbers revealed by analyses of variance analogous to those conducted for inspiratory pressures described previously: For pressures generated at FRC, $F(8,224)=50.74$, and at TLC, $F(8,224)=54.77$. The effects for age were not reliable.

\section{Lung Volume Changes}

Older subjects expelled less air than the younger subjects prior to the production of static inspirations at residual volume. The mean volume change exhibited by older individuals ( 0.60 liter) was reliably less than that exhibited by younger individuals (1.25 liters) $[t(28)=$ $5.87, p<.05]$.

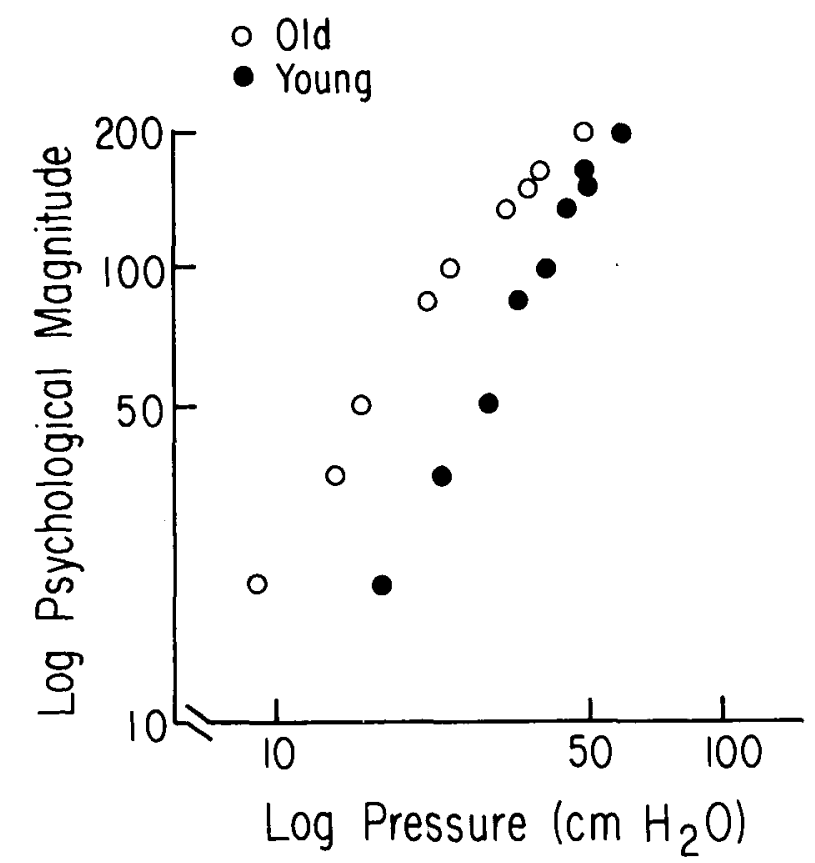

Figure 2. Relationship between psychological magnitude assigned by the experimenter and static inspirations at RV, adjusted for measured relaxation pressures, produced by young and old subjects. Coordinates are logarithmic. 


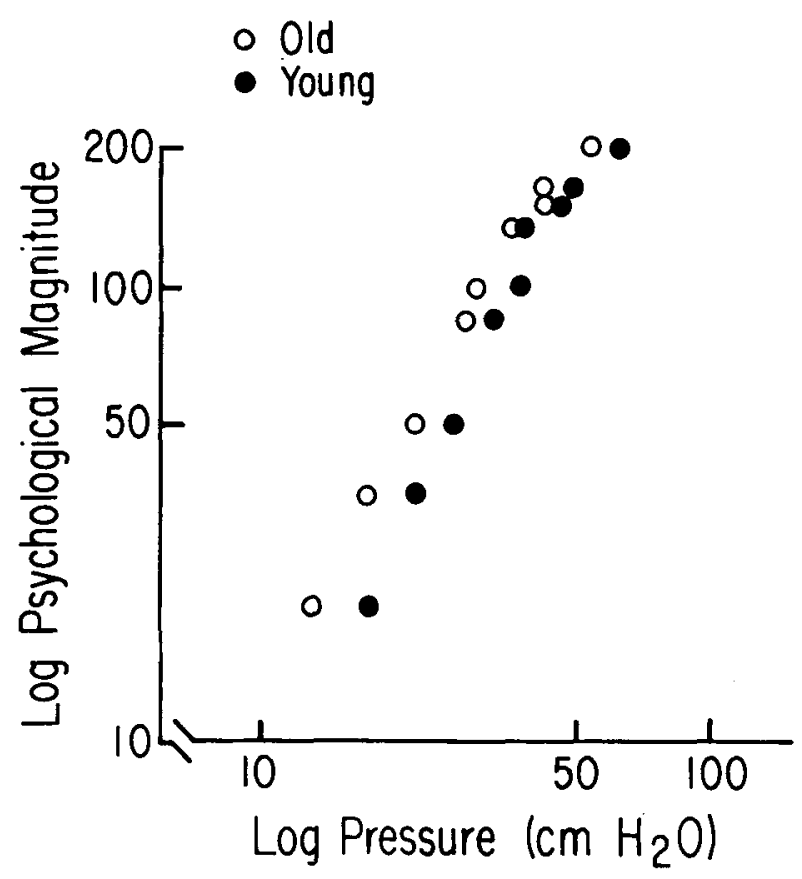

Figure 3. Relationship between psychological magnitude assigned by the experimenter and static expirations at FRC produced by young and old subjects. Coordinates are logarithmic.

No differences in the volume of air inspired prior to the production of static expirations at TLC as a function of age were observed. The mean volume change exhibited by older subjects ( 2.72 liters) was not different from that exhibited by younger subjects (3.04 liters) $[t(28)=1.38$, $p>$.05].

\section{DISCUSSION}

We have shown that exponents for respiratory muscle force for a range of static inspiratory and expiratory tasks are comparable in young and old adults. The finding that young and old subjects do not differ in their exponents for inspiratory force generated by actively contracting muscles at the end of normal expiration is consistent with the results of Tack et al. (1983). Exponents computed for both young and old groups (1.95 and 1.51, respectively) are comparable in degree to those reported previously (about 1.7) and are consistent with previous observations that perception of static inspirations is related directly to tension generated by actively contracting respiratory muscles (Altose et al., 1982; Tack et al., 1983; Zechman \& Wiley, 1986).

It seems likely that exponents for respiratory muscle forces are independent of whether such forces are generated by inspiratory or expiratory muscle groups, but few studies of respiratory sensation have examined perceptual function on both types of tasks. In one study, exponents were comparable for forces generated by respira- tory muscles overcoming loads added to inspiration or to expiration (Muza, McDonald, \& Zechman, 1984).

It seems likely also that exponents for respiratory muscle forces are independent of the lung volume at which forces are generated. The lack of difference between exponents for static inspirations scaled at FRC and those scaled at RV complements previous findings that muscle forces generated at FRC during magnitude scaling were indistinguishable from muscle forces generated during subsequent force reproduction at FRC minus 1 liter (Altose et al., 1982). The similarity of exponents computed for young subjects for static expirations produced at FRC and at TLC in the present study are comparable to those reported previously (1.59 and 1.58 , respectively) (Bakers \& Tenney, 1970).

Exponents for static respiratory muscle forces are similar to those obtained for static handgrip forces (1.6) as well as other effort-dependent tasks (e.g., bicycle ergometer, 1.6) (Jones, 1986). It has been hypothesized that collateral motor command signals mediate the sense of effort in these tasks (Jones, 1986). Such a hypothesis has also been advanced for respiratory sensations (e.g., Gandevia, Killian, \& Campbell, 1981). The possible effects of collateral motor command signals on the perceived magnitude of respiratory sensations, however, remain largely circumstantial (Gandevia, 1986; Harver, Tenney, \& Baird, 1986; Killian \& Campbell, 1985).

Many researchers have documented difficulties in obtaining reliable estimates of relaxation pressures, espe-

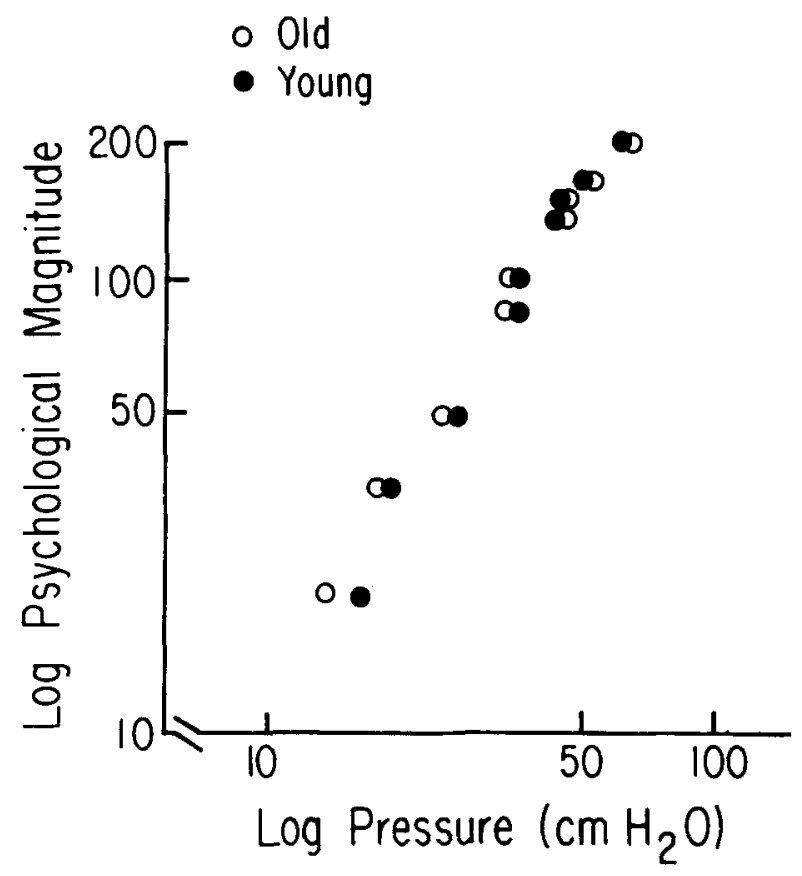

Figure 4. Relationship between psychological magnitude assigned by the experimenter and static expirations at TLC, adjusted for measured relaxation pressures, produced by young and old subjects. Coordinates are logarithmic. 
cially at low lung levels in naive subjects (Knowles, Hong, \& Rahn, 1959; Turner, Mead, \& Wohl, 1968). The absolute relaxation pressures recorded in the present study were only about a third as large as those recorded in trained subjects. To accommodate these discrepancies, mean values for relaxation pressures at RV and at TLC reported previously (Knowles et al., 1959; Rahn, Otis, Chadwick, \& Fenn, 1946; Turner et al., 1968) were used to adjust peak pressure to reflect that due only to actively contracting respiratory muscles. Using these adjusted values, no differences in exponents for production of muscle forces at RV, and TLC, were observed between groups.

\section{REFERENCES}

Altose, M. D., Dimarco, A. F., Gottfried, S. B., \& Strohl, K. P. (1982). The sensation of respiratory muscle force. American Review of Respiratory Disease, 126, 807-811.

Altose, M. D., Leitner, J., \& Cherniack, N. S. (1985). Effects of age and respiratory efforts on the perception of resistive ventilatory loads. Journal of Gerontology, 40, 147-153.

Bakers, J. H. C. M., \& TeNnEY, S. M. (1970). The perception of some sensations associated with breathing. Respiration Physiology, 10, 85-92.

Chernack, R. M. (1983). Mechanics of breathing. Seminars in Respiratory Medicine, 4, 171-182.

Cherniack, R. M., \& Brown, E. (1965). A simple method for measuring total respiratory compliance: Normal values for males. Journal of Applied Physiology, 20, 87-91.

Gandevia, S. C. (1986). Muscle weakness and the power law [Letter to the editor]. American Review of Respiratory Disease, 134, 844-845.

Gandevia, S. C., Killian, K. J., \& Campbell, E. J. M. (1981). The effect of respiratory muscle fatigue on respiratory sensations. Clinical Science, 60, 463-466.

Harver, A., TenNey, S. M., \& Baird, J. C. (1986). A cautionary note on the interpretation of the power law for respiratory effort. American Review of Respiratory Disease, 133, 341-342.

Jones, L. A. (1986). Perception of force and weight: Theory and research. Psychological Bulletin, 100, 29-42.

Killian, K. J., Bucens, D. D., \& Campbell, E. J. M. (1982). Effect of breathing patterns on the perceived magnitude of added loads to breathing. Journal of Applied Physiology: Respiration, Environment, \& Exercise Physiology, 52, 578-584.
Killian, K. J., \& Campbell, E. J. M. (1985). Dyspnea. In C. Roussos \& P. T. Macklem (Eds.), The thorax (Part B, pp. 787-828). New York: Marcel Dekker.

Knowles, J. H., Hong, S. K., \& RAHN, H. (1959). Possible errors using esophageal balloon in determination of pressure-volume characteristics of the lung and thoracic cage. Journal of Applied Physiology, 14, 525-530.

Kory, R. S., Callahan, R., Boren, H. G., \& Syner, J. C. (1961). The Veterans Administration-Army cooperative study of pulmonary function: I. Clinical spirometry in normal men. American Journal of Medicine, 30, 243-258.

MAHLER, D. A. (1983). Pulmonary aspects of aging. In S. R. Gambert (Ed.), Contemporary geriatric medicine (Vol. 1, pp. 45-85). New York: Plenum.

Mittman, C., Edelman, N. H., Norris, A. H., Shock, N. W. (1965). Relationship between chest wall and pulmonary compliance and age. Journal of Applied Physiology, 20, 1211-1216.

Muza, S. R., McDonald, S., \& Zechman, Z. W. (1984). Comparison of subjects' perception of inspiratory and expiratory resistance. Journal of Applied Physiology: Respiration, Environment \& Exercise Physiology, 56, 211-216.

Rahn, H., Otis, A. B., Chadwick, L. E., \& Fenn, W. O. (1946). The pressure-volume diagram of the thorax and lung. American Journal of Physiology, 146, 161-178.

STEVENS, S. S. (1975). Psychophysics: Introduction to its perceptual, neural, and social prospects. New York: Wiley.

TACK, M., Altose, M. D., \& Cherniack, N. S. (1981). Effect of aging on respiratory sensations produced by elastic loads. Journal of $A p^{-}$ plied Physiology: Respiration, Environment, \& Exercise Physiology, 50, 844-850.

TaCk, M., Altose, M. D., \& Cherniack, N. S. (1982). Effect of aging on the perception of resistive ventilatory loads. American Review of Respiratory Disease, 126, 463-467.

Tack, M., Altose, M. D., \& Cherniack, N. S. (1983). Effects of aging on sensation of respiratory force and displacement. Journal of Applied Physiology: Respiration, Environment, \& Exercise Physiology, 55, 1433-1440.

Turner, J. M., Mead, J., \& WoHL, M. E. (1968). Elasticity of human lungs in relation to age. Journal of Applied Physiology, 25, 664-671.

Zechman, F. W., \& WiLEY, R. L. (1986). Afferent inputs to breathing: Respiratory sensation. In A. P. Fishman (Ed.), Handbook of physiology (Sec. 3, Vol. 2, Part 1, pp. 449-474). Bethesda, MD: American Physiological Society.

(Manuscript received December 15, 1986; revision accepted for publication January 18, 1987.) 